\title{
Cobalamin deficiency during treatment of pediatric precursor B-cell lymphoblastic leukemia
}

\author{
Hiromi Kinoshita $^{1}$, Atsuko Watanabe ${ }^{1}$, Yoshitada Taji ${ }^{1}$, Moe Yoshimura ${ }^{1}$, Atsuhiko Ota ${ }^{1}$, \\ Takashi Fukushima ${ }^{1}$, Ryuhei Tanaka ${ }^{1}$, and yasuhiro ebihara ${ }^{1}$ \\ ${ }^{1}$ Saitama Medical University International Medical Center
}

June 1, 2021

Cobalamin deficiency during treatment of pediatric precursor B-cell lymphoblastic leukemia

Hiromi Kinoshita ${ }^{1)}$, Atsuko Watanabe ${ }^{2)}$, Yoshitada Taji ${ }^{1)}$, Moe Yoshimura ${ }^{2)}$, Atsuhiko Ota ${ }^{2)}$, Takashi Fukushima $^{2)}$, Ryuhei Tanaka ${ }^{2)}$, Yasuhiro Ebihara ${ }^{1,3)}$

1) Clinical Laboratory, ${ }^{2)}$ Department of Pediatric Hematology/Oncology, ${ }^{3)}$ Department of Laboratory Medicine, Saitama Medical University International Medical Center, Saitama, Japan

Corresponding author: Yasuhiro Ebihara, MD, Department of Laboratory Medicine, Saitama Medical University International Medical Center, 1397-1 Yamane, Hidaka-shi, Saitama 350-1298, Japan

TEL: +81-42-984-4384; FAX: +81-42-984-4384E-mail:ebihara@saitama-med.ac.jp

Word count

Main text: 500

Figure: 1

Supplementary Table: 1

Running title: Cobalamin deficiency in pediatric BCP-ALL

Keywords: cobalamin deficiency, pediatric B-cell precursor acute lymphoblastic leukemia, erythroid dysplasia, nutrition

To the editor,

There has been considerable progress in supportive therapy and care strategies for patients with cancer, but nutritional problems may still arise during treatment ${ }^{1}$. Herein, we report on a 4 -year-old boy who was diagnosed with B-cell precursor acute lymphoblastic leukemia (BCP-ALL). Complete remission was achieved and maintained. Eight months later, he underwent late intensification treatment, which contained three cycles of methotrexate $500 \mathrm{mg} / \mathrm{m}^{2}$. BM examination scheduled before he went into the maintenance phase showed erythroid dominance and apparent dysplasia, including megaloblastic change, multinuclearity, karyorrhexis, and nuclear budding, and a few Howell-Jolly bodies were noted in the cytoplasm of some erythroid cells (Figure 1). Myelodysplastic syndrome (MDS) was suspected because he had received several anti-leukemic drugs that could induce treatment-related MDS. These morphological changes are seen in folate and/or cobalamin deficiency, which might be mistaken for MDS or acute leukemia ${ }^{2,3}$. The laboratory investigations (Table S1) revealed a decreased serum cobalamin level of $145 \mathrm{pg} / \mathrm{mL}$, indicating that the erythroid dysplasia was due to cobalamin deficiency. Oral replacement therapy of mecobalamin, $500 \mu \mathrm{g} /$ day, was started. Ten days later, BM examination revealed that the erythroid dysplasia had mostly disappeared. The final diagnosis 
was erythroid dysplasia caused by cobalamin deficiency. The patient subsequently underwent maintenance treatment and has remained in CR.

A characteristic feature of cobalamin deficiency is nuclear-cytoplasm asynchrony in erythroid maturation, whereby maturation of the nucleus is delayed relative to that of the cytoplasm because cobalamin is essential for DNA synthesis ${ }^{4,5}$. Cobalamin deficiency is very rare in childhood, and is seen mainly in children with inadequate intake, breast-feeding infants with a cobalamin-deficient mother, and those with congenital malabsorption ${ }^{4,5}$. The occurrence of cobalamin deficiency in the middle of the ALL treatment is extremely rare for the pediatric patients who can take orally and developing normally. In pediatric ALL patients, the existence of cobalamin deficiency before treatment was reported ${ }^{6,7}$, but there is almost no report about cobalamin deficiency during treatment. We could not find any factors which might induce cobalamin deficiency. However, it was possible that the repeated episodes of BM suppression, the recovery from which required more cobalamin for maturation of blood cells, and accumulation of anorexic episodes a caused by administration of anti-leukemic drugs and treatment complications might have led to a gradual decrease in his serum cobalamin level, culminating in cobalamin deficiency. Cobalamin deficiency requires parenteral or oral replacement therapy ${ }^{4,8}$. It is reported that response to replacement is rapid (within 5 days) and megaloblastic anemia can be corrected in 6-8 weeks ${ }^{4,5}$. However, the erythroid dysplasia in BM resolved almost completely after 10 days of cobalamin replacement, and improvement in both peripheral blood (Table S1) and BM were clearly achieved in our patient. Although there have been reports of improved laboratory data in peripheral blood ${ }^{2,4,5}$, there are few descriptions on findings in BM ${ }^{9}$. Although there has been considerable progress in supportive therapy and care strategies for children with cancer, their nutritional status should be monitored carefully during treatment.

\section{Authors' contributions}

H.K., A.W., R.T., and Y.E. designed the study; A.W., M.Y., A.O., T.F., and R.T. treated the patient; H.K., and Y.T. performed the laboratory examinations; and H.K., A.W., R.T., and Y.E. wrote the paper.

\section{Conflicts of interest}

The authors declare no conflicts of interest relevant to this article.

\section{References}

1. Deluche E, Girault S, Jesus P, et al. Assessment of the nutritional status of adult patients with acute myeloid leukemia during induction chemotherapy. Nutrition. 2017;41:120-125.

2. Kim M, Lee SE, Park J, et al. Vitamin B(12)-responsive pancytopenia mimicking myelodysplastic syndrome. Acta Haematol.2011;125(4):198-201.

3. Konda M, Godbole A, Pandey S, Sasapu A. Vitamin B12 deficiency mimicking acute leukemia. Proc (Bayl Univ Med Cent).2019;32(4):589-592.

4. Stabler SP. Clinical practice. Vitamin B12 deficiency. N Engl J Med. 2013;368(2):149-160.

5. Green R, Allen LH, Bjorke-Monsen AL, et al. Vitamin B12 deficiency. Nat Rev Dis Primers. 2017;3:17040.

6. Tandon S, Moulik Nr Fau - Kumar A, Kumar A Fau - Mahdi AA, Mahdi Aa Fau - Kumar A, Kumar A. Effect of Pre-treatment Nutritional Status, Folate and Vitamin B12 Levels on Induction Chemotherapy in Children with Acute Lymphoblastic Leukemia. Indian Pediatr. 2015;52(0974-7559 (Electronic)):385-389.

7. Köse V, Bilir ÖA, Kara A, Özbek NY, Yaralı HN. The impact of pretreatment serum cobalamin and folate levels on complications and peripheral blood recovery during induction chemotherapy of leukemia: a cross-sectional study. Supportive Care in Cancer.2021;29(4):2225-2230.

8. Sezer RG, Akoğlu HA, Bozaykut A, Özdemir GN. Comparison of the efficacy of parenteral and oral treatment for nutritional vitamin B12 deficiency in children. Hematology. 2018;23(9):653-657. 
9. Parmentier S, Meinel J, Oelschlaegel U, et al. Severe pernicious anemia with distinct cytogenetic and flow cytometric aberrations mimicking myelodysplastic syndrome. Ann Hematol.2012;91(12):1979-1981.

\section{Figure legend}

Figure 1. Morphological features in bone marrow. Bone marrow aspirate showing erythroid dysplasia (MayGrunwald-Giemsa staining, original magnification $\times 1,000)$, megaloblastic change with nuclear cytoplasmic asynchrony in erythroblasts (a-d, solid arrows), multinuclear erythroblasts (a, c, d, dashed arrows), HowellJolly bodies (b, c, d, triangles), and nuclear budding (b, asterisk).

FIGURE 1

a)

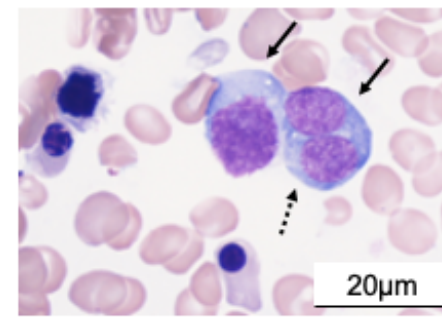

c)

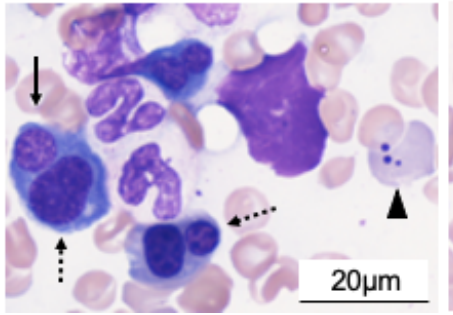

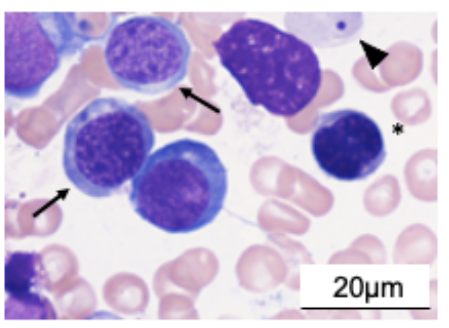

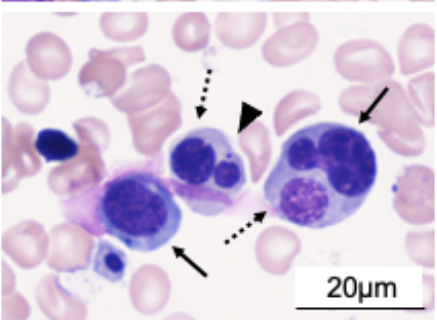

b)

d) 